\section{Lagerung in Neutralstellung - bequemer aber auch besser?}

\begin{abstract}
Die Lagerung schwer immobiler Patienten soll möglichst bequem sein und Komplikationen wie Dekubitus, Kontrakturen und Pneumonien verhindern. Allerdings gibt es in Bezug auf die Effektivität von Lagerungsmethoden bislang nur wenig Evidenz. Daher ist bislang die erforderliche Frequenz von Umlagerungen und ob bzw. welche konsequent angewendete Lagerung die funktionelle Erholung unterstützen kann, unklar.
\end{abstract}

॥ Durch diverse, insbesondere neurologische Erkrankungen leiden viele Menschen unter schwerer Immobilität und benötigen über einen langen Zeitraum eine therapeutische Lagerung. Dies ist ein wichtiger Teil der pflegetherapeutischen Routine sowohl in der Akutversorgung als auch während des rehabilitativen Prozesses.

In der vorliegenden Studie von 2015 wurde die Effektivität zweier Lagerungsformen hinsichtlich der passiven Beweglichkeit der Hüften und der Schultern (PROM, "passive range of motion") untersucht und das Wohlbefinden der Patienten dabei verglichen. Als Lagerungskonzepte kamen die herkömmliche Lagerung (KON, ,,konventionell“), bei der alle zwei bis drei Stunden ein Wechsel zwischen unterschiedlichen Positionen wie Rückenlage, Bauchlage oder Seitenlagen erfolgt (mit Hilfe von Lagerungsmaterial), und die Lagerung in Neutralstellung (LiN) zum Einsatz. Bei diesem Lagerungskonzept spielt die Stellung der Körperabschnitte zueinander eine entscheidende Rolle. Muskelgruppen sollen weder verkürzt noch überdehnt und paretische Körperabschnitte stabilisierend unterstützt werden. Die Gelenke werden so neutral wie möglich eingestellt. Für die Lagerung in Neutralstellung wird mehr Lagerungsmaterial benötig. Außerdem ist diese Lagerungsform aufwändiger als die herkömmliche Lagerung.

Um die Effektivität der beiden Lagerungsformen zu untersuchen, wurden in dieser prospektiven, multizentrischen (22 Zentren für Frührehabilitation und geriatrische Rehabilitation, Schlaganfall-, Intensiv- und Wachkomastationen, Pflegeheime in Deutschland und Österreich), Untersucher-verblindeten, randomisierten und kontrollierten Studie 218 nicht gehfähige, vorwiegend neurologische Patienten eingeschlossen. Einschlusskriterien waren ein Mindestalter von 18 Jahren und eine zentrale Parese mit schwerer Behinderung der Mobilität.

\section{Kommentar}

Das Ziel der vorliegenden Untersuchung war es, die Effektivität der herkömmlichen Lagerung mit der Lagerung in Neutralstellung bei Patienten mit schwerer Immobilität zu vergleichen. Mit der vorliegenden Forschungsarbeit konnte gezeigt werden, dass durch die Lagerung in Neutralstellung die passive Beweglichkeit von Hüften und Schultern vergrößert werden kann und die Lagerung in Neutralstellung dabei vom Patienten als deutlich bequemer empfunden wird als eine herkömmliche Lagerung.

Die Studie erreicht aufgrund ihrer multizentrischen Ausrichtung, der Randomisierung der Probanden, der Verblindung der Untersucher und des Vorhandenseins einer Kontrollgruppe eine hohe wissenschaftliche Evidenz. Die sehr eindeutigen Ergebnisse müssten eigentlich in allen Einrichtungen, in denen immobile Patienten versorgt, also auch regelmäßig gelagert werden, zum Nachbzw. Umdenken angeregt haben. Obwohl die Einführung eines neuen Lagerungskonzeptes sehr zeitaufwendig und kostenintensiv ist, sollten so schnell wie möglich noch weitere, ebenfalls von hoher wissenschaftlicher Güte geprägte Studien durchgeführt werden, die beispielsweise den Einfluss auf die Entstehung von Dekubiti oder die Langzeiteffekte der Lagerung in Neutralstellung untersuchen.
}

Diese Patienten wurden zufällig einer Lagerungsform (LiN: $\mathrm{n}=105, \mathrm{KON}$ : $\mathrm{n}=$ 113) zugewiesen und innerhalb jedes Lagerungskonzeptes in fünf Positionen stratifiziert.

\section{Verbesserung in der PROM}

Die passive Beweglichkeit der Hüften und der Schultern wurde vor und nach zwei Stunden des Liegens (entweder in LiN oder in KON) mit einem Standardwinkelmesser vorgenommen. Das Wohlbefinden der Patienten wurde nach der Lagerung bei den kommunikationsfähigen Patienten mit einer dreistufigen Skala („gut“, „mittel“, „schlecht“) erfragt.

Nach der statistischen Analyse mit SAS 9.3 erhielten die Forscher um H. Pickenbrock folgende Ergebnisse: Die Patienten der LiN-Gruppe zeigten eine statistisch signifikante Verbesserung der PROM der Hüften nach zwei Stunden Lagerung verglichen zu der KONGruppe. Außerdem führten insgesamt niedrigere PROM-Ausgangswerte im Mittel zu einer stärkeren Verbesserung der passiven Beweglichkeit der Hüften. Ähnliche Effekte zeigten sich auch bei der Schulterbeweglichkeit. Die Frage nach dem Komfort konnten nur 138 Patienten beantworten (80 Patienten waren nicht kommunikationsfähig): Der Komfort wurde für die Lagerung in Neutralstellung als signifikant besser als für die herkömmliche Lagerung bewertet.

\section{Quelle}

Pickenbrock H, Ludwig VU, Zapf A, Dressler $D$. Conventional versus neutral positioning in central neurological disease - a multicenter randomized controlled trial. Dtsch Arztebl Int 2015; 112: 35-42

Autorenkontakt:

Referiert und kommentiert von Oliver Ludwig, examinierter

Gesundheits- und Krankenpfleger und Diplompflegepädagoge, Jena.

E-Mail: oliver_ludwig@gmx.de 